\title{
Durable response to anti-PD-1 immunotherapy in epithelioid angiomyolipoma: a report on the successful treatment of a rare malignancy
}

Michael Lattanzi ${ }^{1}$, Fang-Ming Deng ${ }^{2,3,5}$, Luis A. Chiriboga ${ }^{2}$, Alisa N. Femia ${ }^{4}$, Shane A. Meehan²,4 Gopa lyer ${ }^{6}$, Martin H. Voss ${ }^{6}$, Yuliya Sundatova ${ }^{5,7}$, William C. Huang ${ }^{3,5}$ and Arjun V. Balar ${ }^{1,5,7^{*}}$

\begin{abstract}
Background: Malignant angiomyolipoma is an uncommon tumor of the class of perivasciular epithelioid cell neoplasms (PEComas). These tumors are characteristically driven by deleterious mutations in the tumor suppressors TSC1 and TSC2, whose gene products typically act to inhibit mTOR. There are several cases of malignant angiomyolipoma which exhibit transient responses to mTOR inhibitors, forming the basis of current practice guidelines in malignant PEComa. However the tumors ultimately acquire resistance, and there is no well-established second-line option. Despite the increasing prevalence of immunotherapy across a wide range of solid tumors, little is known about the immune infiltrate and PD-L1 expression of angiomyolipoma. Furthermore, there is no reported case on the treatment of malignant angiomyolipoma with an immune checkpoint inhibitor.

Case presentation: A 38 year-old man presented with gross hematuria and was diagnosed with renal epithelioid angiomyolipoma. Despite surgical resection, the tumor recurred and metastasized. Targeted genomic sequencing revealed a deleterious mutation in TSC2, and the patient was treated with the mTOR inihbitor everolimus. The patient went on to have a partial response but ultimately progressed. He was then treated with the anti-PD-1 immune checkpoint inhibitor nivolumab, and achieved a durable near-complete response which is ongoing after two years of treatment. Immunohistochemical staining of tumor tissue revealed strong PD-L1 expression and a brisk T-cell infiltrate.

Conclusions: We report on the first durable systemic treatment of malignant epithelioid angiomyolipoima with the use of PD-1 antibody nivolumab. Given the absence of prospective clinical trials in this exceedingly rare disease, particularly in the second-line setting, immune checkpoint inhibitors like nivolumab should be considered.
\end{abstract}

Keywords: Immunotherapy, Angiomyolipoma, PEComa, Nivolumab, PD-1, PD-L1, Tuberous sclerosis, TSC2, mTOR, Everolimus

\footnotetext{
* Correspondence: Arjun.Balar@nyumc.org

${ }^{1}$ Department of Medicine, NYU Langone Health, New York, NY, USA

${ }^{5}$ Laura and Isaac Perlmutter Cancer Center, NYU Langone Health, New York,

NY, USA

Full list of author information is available at the end of the article
}

(c) The Author(s). 2018 Open Access This article is distributed under the terms of the Creative Commons Attribution 4.0 International License (http://creativecommons.org/licenses/by/4.0/), which permits unrestricted use, distribution, and reproduction in any medium, provided you give appropriate credit to the original author(s) and the source, provide a link to the Creative Commons license, and indicate if changes were made. The Creative Commons Public Domain Dedication waiver (http://creativecommons.org/publicdomain/zero/1.0/) applies to the data made available in this article, unless otherwise stated. 


\section{Background}

Angiomyolipomas (AMLs) are neoplasms thought to arise from pericites, hence belonging to the family of perivasciular epithelioid cell neoplasms (PEComas). Though associated with tuberous sclerosis complex (TSC), most AMLs arise de novo, in the absence of germline TSC1 or TSC2 mutations [1]. There exist two well-described histologic variants: classical and epithelioid. Although the vast majority of AMLs are benign, a minority of epithelioid AML (EAML) may become malignant and have been reported to metastasize [2]. The exact prevalence of malignant EAML is not well characterized; however, it likely falls below 1:300,000 [3, 4]. Given its rarity, there is no established treatment for unresectable or metastatic EAML.

TSC is a genetic syndrome characterized by multisystem tumor development, including renal angiomyolipoma. Most patients harbor pathogenic germline loss-of-function mutations in TSC1 or TSC2 [5], whose wild-type gene products inhibit mammalian target of rapamycin (mTOR) complex 1 (mTORC1) [6, 7]. This observation prompted a phase III placebo-controlled trial demonstrating significant tumor regression of TSC-associated AML with everolimus [8], a United States Food and Drug Administration (FDA)-approved allosteric inhibitor of mTORC1. Somatic mutations in TSC1 and TSC2 also contribute to tumor growth via unopposed mTOR signaling, and sporadic AML is similarly characterized by somatic loss-of-function alterations in TSC2 [9]. Multiple reports detail responses to mTOR inhibitors among tumors harboring TSC1 or TSC2 mutations [7, 10], including PEComa, not otherwise specified [11], and sporadic AML [12], though DNA sequencing was not reported. Based on these responses, clinical practice guidelines for malignant PEComa currently emphasize the use of mTOR inhibitors such as everolimus [13]. However, despite an initial response to rapalog therapy, virtually all patients ultimately develop progressive disease, and there is no well-established second-line treatment.

Nivolumab is a fully humanized monoclonal IgG4 antibody that targets the programmed death 1 (PD-1) receptor, an immune checkpoint expressed on exhausted effector $\mathrm{T}$ lymphocytes, and prevents binding by its activating ligand PD-L1, leading to reinvigoration of anti-tumor immunity [14]. Nivolumab is FDA-approved for melanoma, renal cell carcinoma, and urothelial bladder cancer, among other solid tumors. Although tumor PD-L1 expression is associated with response [15], no biomarker of response has been rigorously validated. Additionally, immune checkpoint inhibitors are associated with the development and/or exacerbation of autoimmunity [15], and such immune-related toxicities may correlate with enhanced clinical efficacy [16].

Given the lack of data concerning the treatment of this rare cancer, we report a case of metastatic EAML harboring a deleterious TSC2 mutation. The patient exhibited a transient response to everolimus, but ultimately progressed. He subsequently achieved a significant and durable response to nivolumab. To the best of our knowledge, this is the first report on the treatment of malignant EAML with immunotherapy.

\section{Case presentation}

A 38 year-old man with vitiligo and hypothyroidism initially presented in 2011 with gross hematuria. Diagnostic imaging (Fig. 1a) revealed a 6-cm renal mass concerning for malignancy, for which he underwent a right radical nephrectomy at the recommendation of his treating urologic oncologist (WCH). Gross pathology (Fig. 1b) revealed a $6 \times 5-\mathrm{cm}$ encapsulated hilar mass with hemorrhage and central necrosis. The mass was limited to the renal parenchyma, without evidence of renal sinus or vascular invasion, and surgical margins were negative for tumor cells. Histologic sections (Fig. 1c) demonstrated sheets of epithelioid cells with sarcomatoid and rhabdoid features as well as round, polygonal cells with pleomorphic nuclei and prominent nucleoli. Mitotic figures were visualized at a rate of approximately three per high-powered field. Immunohistochemical staining (Fig. 1d-e) revealed tumor cell positivity for: HMB45, melan-A, carbonic anhydrase IX, and to a lesser extent, Cam5.2, vimentin and SMA (cytoplasmic), and negativity for: EMA, keratins (AE1/3), CK7, CK20, P63, Pax-2, AMACAR, S-100, and CD10. Based on these histopathologic features, the patient was diagnosed with primary EAML.

The patient had an uneventful course for the next 3 years until April, 2014, when surveillance imaging detected an asymptomatic 13-cm renal fossa mass for which he underwent repeat surgical resection. Surgical pathology confirmed recurrent EAML, again with negative margins. The patient's tumor recurred again in October, 2014, prompting a third surgical resection. Pathologic evaluation this time demonstrated indeterminate margins, prompted referral to medical oncology for further management.

December, 2014 surveillance imaging obtained by the treating medical oncologist (AVB) demonstrated new retroperitoneal and pelvic implants consistent with metastatic EAML. The patient's tumor DNA was subjected to FoundationOne $^{\bullet}$ targeted next-generation sequencing [17], which revealed four oncogenic alterations: truncating mutations in TP53 and APC, a frameshift mutation in $A T R X$, and a deletion in TSC2, specifically, TSC2 H1746_R1751del, which has been reported both as a somatic variant in AML [18] and as a germline mutation in TSC [19]. Of note, the FoundationOne ${ }^{\varpi}$ assay demonstrated no genomic alterations in the four genes encoding key DNA mismatch repair proteins: $M S H 2$, MSH6, PMS2, or MLH1. 

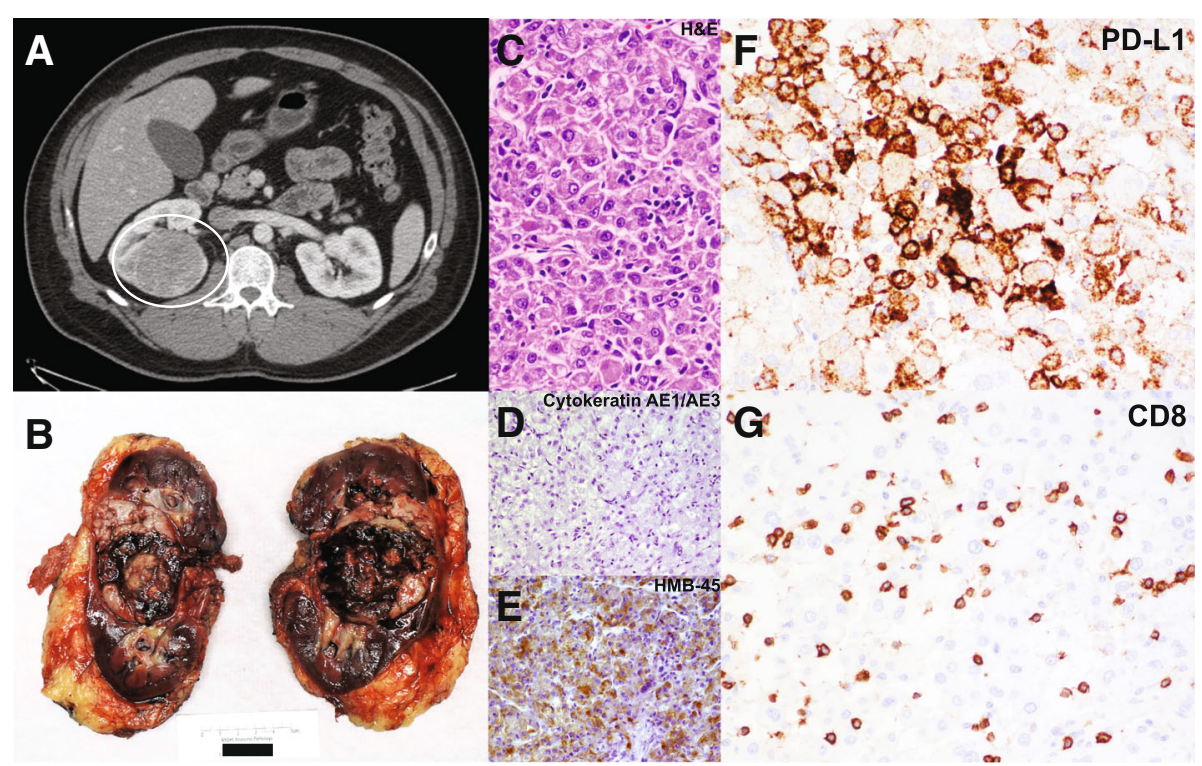

Fig. 1 a CT Urogram demonstrating the primary right renal mass, (b) Gross pathology demonstrating the resected perihilar tumor with central necrosis, (c) H\&E stain demonstrating angiomyolipoma with a substantial epithelial component, (d) Immunohistochemical stain negative for cytokeratin AE1/AE3, (e) Immunohistochemical stain positive for HMB-45, (f) Immunohistochemical stain positive for PD-L1 (> 50\% of cells), (f) Immunohistochemical stain positive for T lymphocyte marker CD8

Based on the TSC2 deletion, the patient was initiated on everolimus in January, 2015. Imaging at 3 months (Fig. 2) demonstrated marked decrease in size of the majority of the soft tissue masses throughout the right nephrectomy bed, retroperitoneum, and mesentery, and no new sites of disease. The patient remained clinically asymptomatic for 8 months, until he noted unintended weight loss in September, 2015. Imaging demonstrated slight enlargement of the dominant right renal fossa mass (Fig. 2), which in the context of progressive anemia, was interpreted as disease progression. Everolimus was discontinued, and the patient was referred for a treatment-directed biopsy for consideration of a clinical trial.

He underwent a biopsy of the dominant $6 \mathrm{~cm}$ retroperitoneal mass, from which DNA was isolated and subjected to paired tumor-germline next-generation sequencing via MSK-IMPACT [20], which confirmed the absence of a TSC2 germline mutation. However, no new somatic variants were identified to explain the tumors'

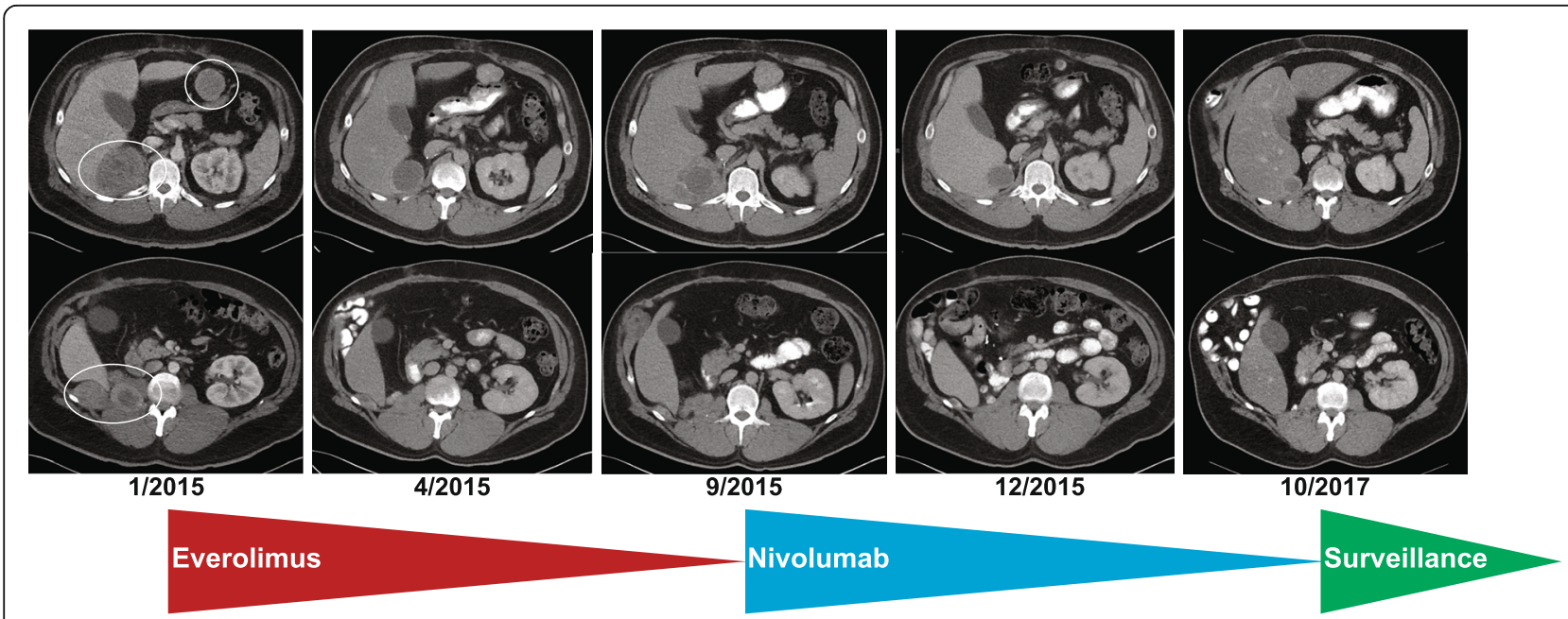

Fig. 2 Schematic representation of radiographic tumor burden over time indicating (left-to-right): baseline pre-everolimus scan, best response to everolimus, progression on everolimus and baseline pre-nivolumab scan, initial post-nivolumab response, and continued response at the time of nivolumab discontinuation 
acquired resistance, and he was not eligible for any clinical trials. Given the well-known activity of anti-PD-1 checkpoint inhibition across a range of advanced solid tumors, including renal cell carcinoma [21], the patient was offered a trial of off-label nivolumab via the Bristol-Myers Squibb Expanded Access program, and he began treatment in October, 2015. After two cycles of nivolumab (administered at $3 \mathrm{mg} / \mathrm{kg}$ IV every 2 weeks), the patient felt well, and resolution of his anemia suggested possible clinical benefit. Imaging after 5 cycles demonstrated responding disease (Fig. 2).

Nivolumab was well-tolerated, with the exception of immune-related exacerbation of pre-existing hypothyroidism (Fig. 3a-b) after 2 months of therapy, and immune-related pruritic cutaneous eruption predominantly within areas of pre-existing vitiligo (Fig. 3c-d), occurring after 18 months of treatment. Over the course of nivolumab therapy, the patient required increasing doses of levothyroxine to maintain a euthyroid state. An archival thyroid ultrasound reveals an enlarged heterogenous thyroid gland suggestive of possible autoimmune thyroiditis. With regard to cutaneous toxicity, the patient was referred to dermatology (ANF), and a skin biopsy was obtained of an involved area on the upper back. Histologic evaluation by the dermatopathologist (SAM) revealed a thin granular layer, confluent parakeratosis with collections of neutrophils, and dilated capillaries throughout the papillary dermis, consistent with psoriasis (Fig. 3e-f).

Although these toxicities were not dose-limiting, the patient had already completed 2 years of therapy [22], and nivolumab was therefore discontinued. The most recent imaging at the time of discontinuation in November, 2017 demonstrates continued response and interval calcification of his intra-abdominal tumors (Fig. 2). Archival tissue derived from the initial surgical resection was analyzed for PD-L1 and CD8 expression via immunohistochemistry (Fig. 1f-g) utilizing a modified Agilent/ Dako 22C3 pharmaDX kit, revealing high PD-L1 expression (>50\% of cells) and a brisk CD8+ T cell infiltrate. Finally, immunohistochemical analysis demonstrated preserved expression of key DNA mismatch repair (MMR) proteins MSH2, MSH6, PMS2, and MLH1 [23], thus confirming MMR proficiency (Fig. 4).

\section{Discussion}

Current approaches to treatment for the sporadic form of recurrent or metastatic EAML are derived from anecdotal reports of efficacy with mTOR inhibitors [11, 24-27]. Furthermore, although Wagner, et al. [11] reported TSC1 loss in PEComa, genomic sequencing of TSC1 and TSC2 was not performed in these cases, as they predate the widespread use of next-generation sequencing, and the histologic subtype (e.g. angiomyolipoma) was not specified. Nonetheless, despite an initial response to rapalog therapy, virtually all tumors ultimately developed resistance. In addition to mTOR inhibitors, tumor responses have been observed with the use of chemotherapy. Cibas, et al. reported a partial response to doxorubicin [28], and others have reported various combinations of cisplatin, cyclophosphamide, dacarbazine, and ifosfamide [29], with modest results and no reported long-term responses.

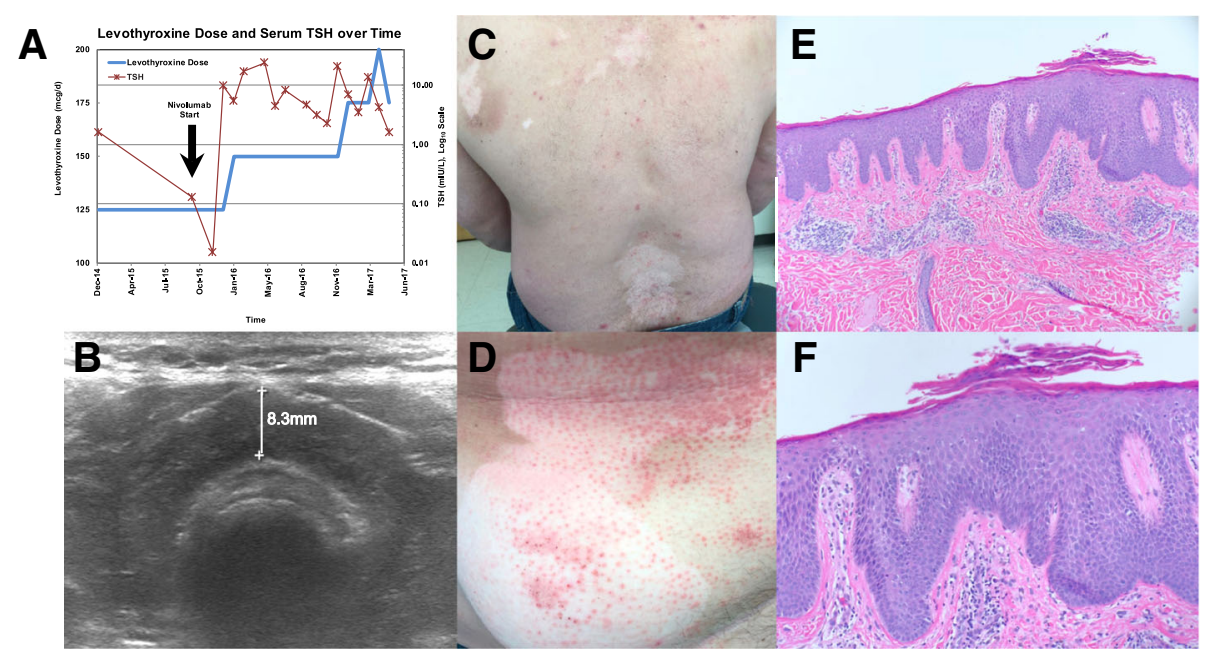

Fig. 3 a Graph of the patient's prescribed daily dose of levothyroxine and serum thyroid stimulating hormone (TSH) over time relative to initiation of nivolumab, (b) Archival thyroid ultrasound demonstrating enlarged, heterogeneous thyroid gland suggestive of chronic thyroiditis, (c) Distribution of psoriasis involving the patient's back, (d) Representative scaly, well-demarcated erythematous papules overlying the right lower abdomen predominantly within an area of vitiligo, (e) Low-resolution and (f) High-resolution histologic sections of cutaneous biopsy with H\&E staining demonstrating a thin granular layer, confluent parakeratosis with collections of neutrophils, and dilated capillaries throughout the papillary dermis, consistent with psoriasis 


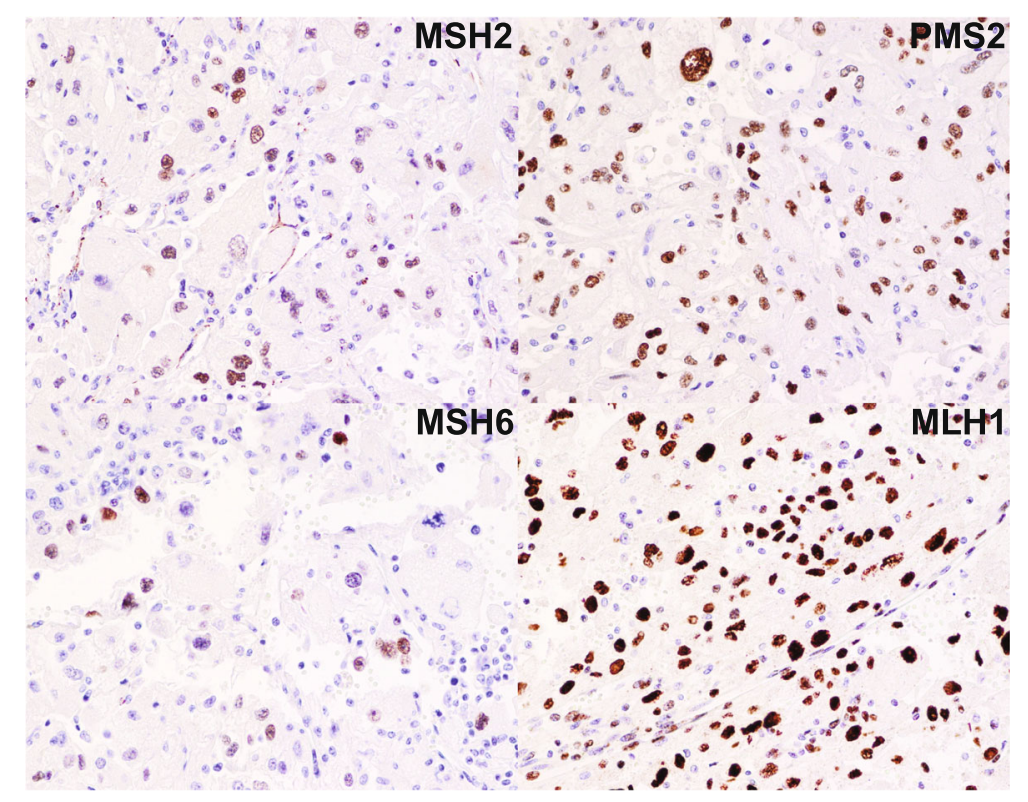

Fig. 4 Immunohistochemical stains for key DNA mismatch repair proteins revealing preserved expression of MSH2, MSH6, PMS2, and MLH1, thereby confirming DNA mismatch repair proficiency

Cumulatively, TSC1 and TSC2 alterations occur in approximately $5 \%$ of all solid tumors, including $4.7 \%$ of the MSK-IMPACT cohort [20] and 5.2\% of the corresponding TCGA cohort [30]. In the COSMIC database [31], TSC2 H1746_R1751del has been previously identified in six tumors, including two renal angiomyolipomas, as reported by Qin, et al. [18]. However, neither of these cases was predominantly epithelioid; neither was reportedly malignant; and one carried a diagnosis of TSC. Of note, genomic sequencing reported by Wagle, et al. identified a gain-of-function mutation in MTOR as the etiology of everolimus resistance in TSC2-mutant thyroid carcinoma. However, no post-treatment alterations were identified to explain the acquired resistance in the present case of EAML.

In contrast to the transient responses to everolimus both in our case and others', we report the first durable response to any agent in this rare malignancy with the use of the PD-1 antibody nivolumab. To the best of our knowledge, this case is the only report on the use of immunotherapy in the management of angiomyolipoma of any primary site or histologic variant. Immunohistochemistry revealed high PD-L1 expression $(>50 \%$ of cells), and brisk cytotoxic T cell infiltrate (Fig. 1f-g). The striking PD-L1 expression in a tumor responsive to anti-PD-1 checkpoint inhibitor immunotherapy is consistent with previous reports supporting a correlation between PD-L1 expression and response to anti-PD-1 and/or anti-PD-L1 immunotherapy. Specifically, in urothelial carcinoma [32] and non-small cell lung adenocarcinoma [33], there is a strong relationship between
PD-L1 expression and response to nivolumab. PD-L1 positivity in this case of nivolumab-responsive EAML may represent a predictive biomarker in this histology as in other cancers, however, this case is the only report of PD-L1 expression in EAML and further study is needed. In melanoma, lymphocytic infiltrate is associated with increased likelihood of response to anti-PD-1 checkpoint inhibitor [34]. The presence of a brisk CD8+ T cell infiltrate suggests intrinsic anti-tumor immunologic activity and may also represent a biomarker of response in EAML. Interestingly, although this patient initiated nivolumab prior to the FDA's histology-independent approval for PD-1 antibodies in DNA mismatch repair deficient (dMMR) tumors, subsequent immunohistochemical analysis demonstrated MMR proficiency, and this patient would not have been a candidate for nivolumab on the basis of MMR status.

Also of interest is our patient's history of pre-existing as well as treatment-related autoimmunity. The development of immune-related adverse events has been correlated to clinical benefit with immunotherapy among melanoma patients, a subset of whom develop vitiligo [16]. In the present patient, the development of antiPD-1-related psoriaform dermatitis and an exacerbation of presumed autoimmune thyroiditis are highly suggestive of a systemic immune activation secondary to anti-PD-1 immunotherapy.

\section{Conclusions}

In this case of malignant EAML - a rare disease with no standard of care - we report a durable near-complete 
response to the anti-PD-1 immune checkpoint inhibitor, nivolumab. This case highlights the cross-histologic efficacy of immune checkpoint inhibition, particularly in tumors with high PD-L1 expression and brisk lymphocytic infiltrate. In the absence of prospective clinical trials, nivolumab should be considered for use in other patients with recurrent or metastatic EAML who have exhausted traditional therapeutic options.

\section{Abbreviations}

AML: Angiomyolipoma; EAML: Epithelioid angiomyolipoma; FDA: Food and Drug Administration; MMR: Mismatch repair; MTOR: Mammalian target of rapamycin; PD-1: Programmed death receptor 1; PD-L1: Programmed death receptor ligand 1; TSC: Tuberous sclerosis complex

\section{Funding}

This study was not supported by any external funding.

\section{Availability of data and materials}

Identifying patient information must remain confidential; however additional data may be available from upon reasonable request at the discretion of the corresponding author.

\section{Authors' contributions}

$\mathrm{ML}$ is a research coordinator for the NYU genitourinary oncology tumor registry and compiled all patient data. FMD is the attending genitourinary pathologist who diagnosed the case. LAC is the director of the immunohistochemistry core facility and performed all correlative staining ANF is the attending dermatologist who performed the cutaneous biopsy. SAM is the attending dermatopathologist who diagnosed the cutaneous toxicity. GI and MHV are medical oncologists who consulted on the case and orchestrated the treatment-directed biopsy and subsequent genomic sequencing. YS is the nurse practitioner who oversaw the patient's medical oncology care. WCH is the attending urologic oncologist who performed all surgical resections. AVB is the attending genitourinary medical oncologist who treated the patient and directed all study-related activities. All authors read and approved the final manuscript.

\section{Ethics approval and consent to participate}

The present study was conducted in accordance with all accepted standards for the ethical conduct of human subjects research. The patient's data and tissue specimens are stored according to NYU IRB-approved protocol s15-01579. The patient provided written informed consent at the time of enrollment.

\section{Consent for publication}

The patient provided verbal and written consent to publish all pertinent demongraphic, clinical, and biologic information. The signed consent form will be made available to the editorial staff upon request.

\section{Competing interests}

$\mathrm{MHV}$ reports consulting fees from Novartis as well as research funding from Genentech and Bristol-Myers Squibb. All other authors report no disclosures.

\section{Publisher's Note}

Springer Nature remains neutral with regard to jurisdictional claims in published maps and institutional affiliations.

\footnotetext{
Author details

${ }^{1}$ Department of Medicine, NYU Langone Health, New York, NY, USA. 2Department of Pathology, NYU Langone Health, New York, NY, USA. ${ }^{3}$ Department of Urology, NYU Langone Health, New York, NY, USA. ${ }^{4}$ Ronald O. Perelman Department of Dermatology, NYU Langone Health, New York, NY, USA. ${ }^{5}$ Laura and Isaac Perlmutter Cancer Center, NYU Langone Health, New York, NY, USA. ${ }^{6}$ Department of Medicine, Memorial Sloan-Kettering Cancer Center, New York, NY, USA. ' Genitourinary Medical Oncology Program, NYU School of Medicine, Laura and Isaac Perlmutter Cancer Center, NYU Langone Health, 160 East 34th Street, 10th Floor, New York, NY 10016, USA.
}

Received: 15 June 2018 Accepted: 18 September 2018

Published online: 01 October 2018

\section{References}

1. Flum AS, Hamoui N, Said MA, Yang XJ, Casalino DD, McGuire BB, et al. Update on the diagnosis and Management of Renal Angiomyolipoma. J Urol. 2016;195(4 Pt 1):834-46.

2. Aydin H, Magi-Galluzzi C, Lane BR, Sercia L, Lopez Jl, Rini Bl, et al. Renal angiomyolipoma: clinicopathologic study of 194 cases with emphasis on the epithelioid histology and tuberous sclerosis association. Am J Surg Pathol. 2009;33(2):289-97.

3. He W, Cheville JC, Sadow PM, Gopalan A, Fine SW, Al-Ahmadie HA, et al. Epithelioid angiomyolipoma of the kidney: pathological features and clinical outcome in a series of consecutively resected tumors. Mod Pathol. 2013; 26(10):1355-64

4. Fujii Y, Ajima J, Oka K, Tosaka A, Takehara Y. Benign renal tumors detected among healthy adults by abdominal ultrasonography. Eur Urol. 1995;27(2): 124-7.

5. Northrup H, Krueger DA. International tuberous sclerosis complex consensus G. tuberous sclerosis complex diagnostic criteria update: recommendations of the 2012 linternational tuberous sclerosis complex consensus conference. Pediatr Neurol. 2013;49(4):243-54.

6. El-Hashemite N, Zhang H, Henske EP, Kwiatkowski DJ. Mutation in TSC2 and activation of mammalian target of rapamycin signalling pathway in renal angiomyolipoma. Lancet. 2003;361(9366):1348-9.

7. Iyer G, Hanrahan AJ, Milowsky Ml, Al-Ahmadie H, Scott SN, Janakiraman M, et al. Genome sequencing identifies a basis for everolimus sensitivity. Science. 2012;338(6104):221.

8. Bissler JJ, Kingswood JC, Radzikowska E, Zonnenberg BA, Frost M, Belousova E, et al. Everolimus for angiomyolipoma associated with tuberous sclerosis complex or sporadic lymphangioleiomyomatosis (EXIST-2): a multicentre, randomised, double-blind, placebo-controlled trial. Lancet. 2013;381(9869): 817-24.

9. Smolarek TA, Wessner LL, McCormack FX, Mylet JC, Menon AG, Henske EP. Evidence that lymphangiomyomatosis is caused by TSC2 mutations: chromosome $16 \mathrm{p} 13$ loss of heterozygosity in angiomyolipomas and lymph nodes from women with lymphangiomyomatosis. Am J Hum Genet. 1998; 62(4):810-5

10. Wagle N, Grabiner BC, Van Allen EM, Amin-Mansour A, Taylor-Weiner A, Rosenberg $\mathrm{M}$, et al. Response and acquired resistance to everolimus in anaplastic thyroid cancer. N Engl J Med. 2014;371(15):1426-33.

11. Wagner AJ, Malinowska-Kolodziej I, Morgan JA, Qin W, Fletcher CD, Vena N, et al. Clinical activity of mTOR inhibition with sirolimus in malignant perivascular epithelioid cell tumors: targeting the pathogenic activation of mTORC1 in tumors. J Clin Oncol. 2010;28(5):835-40.

12. Bissler JJ, McCormack FX, Young LR, Elwing JM, Chuck G, Leonard JM, et al. Sirolimus for angiomyolipoma in tuberous sclerosis complex or lymphangioleiomyomatosis. N Engl J Med. 2008;358(2):140-51.

13. von Mehren M, Randall RL, Benjamin RS, Boles S, Bui MM, Conrad EU 3rd, et al. Soft tissue sarcoma, version 2.2016, NCCN clinical practice guidelines in oncology. J Natl Compr Cancer Netw. 2016;14(6):758-86.

14. Wolchok JD, Kluger H, Callahan MK, Postow MA, Rizvi NA, Lesokhin AM, et al. Nivolumab plus ipilimumab in advanced melanoma. N Engl J Med. 2013;369(2):122-33.

15. Topalian SL, Hodi FS, Brahmer JR, Gettinger SN, Smith DC, McDermott DF, et al. Safety, activity, and immune correlates of anti-PD-1 antibody in cancer. N Engl J Med. 2012;366(26):2443-54.

16. Teulings HE, Limpens J, Jansen SN, Zwinderman AH, Reitsma JB, Spuls PI, et al. Vitiligo-like depigmentation in patients with stage III-IV melanoma receiving immunotherapy and its association with survival: a systematic review and meta-analysis. J Clin Oncol. 2015;33(7):773-81.

17. Frampton GM, Fichtenholtz A, Otto GA, Wang K, Downing SR, He J, et al. Development and validation of a clinical cancer genomic profiling test based on massively parallel DNA sequencing. Nat Biotechnol. 2013;31(11): 1023-31.

18. Qin W, Bajaj V, Malinowska I, Lu X, MacConaill L, Wu CL, et al. Angiomyolipoma have common mutations in TSC2 but no other common genetic events. PLoS One. 2011;6(9):e24919.

19. Roberts PS, Dabora S, Thiele EA, Franz DN, Jozwiak S, Kwiatkowski DJ. Somatic mosaicism is rare in unaffected parents of patients with sporadic tuberous sclerosis. J Med Genet. 2004;41(5):e69. 
20. Zehir A, Benayed R, Shah RH, Syed A, Middha S, Kim HR, et al. Mutational landscape of metastatic cancer revealed from prospective clinical sequencing of 10,000 patients. Nat Med. 2017;23(6):703-13.

21. Motzer RJ, Escudier B, McDermott DF, George S, Hammers HJ, Srinivas S, et al. Nivolumab versus Everolimus in advanced renal-cell carcinoma. N Engl J Med. 2015;373(19):1803-13.

22. Robert C, Schachter J, Long GV, Arance A, Grob JJ, Mortier L, et al. Pembrolizumab versus Ipilimumab in advanced melanoma. N Engl J Med. 2015;372(26):2521-32.

23. Le DT, Durham JN, Smith KN, Wang H, Bartlett BR, Aulakh LK, et al. Mismatch repair deficiency predicts response of solid tumors to PD-1 blockade. Science. 2017;357(6349):409-13.

24. Italiano A, Delcambre C, Hostein I, Cazeau AL, Marty M, Avril A, et al. Treatment with the mTOR inhibitor temsirolimus in patients with malignant PEComa. Ann Oncol. 2010:21(5):1135-7.

25. Gennatas C, Michalaki V, Kairi PV, Kondi-Paphiti A, Voros D. Successful treatment with the MTOR inhibitor everolimus in a patient with perivascular epithelioid cell tumor. World J Surg Oncol. 2012;10:181.

26. Benson C, Vitfell-Rasmussen J, Maruzzo M, Fisher C, Tunariu N, Mitchell S, et al. A retrospective study of patients with malignant PEComa receiving treatment with sirolimus or temsirolimus: the Royal Marsden Hospital experience. Anticancer Res. 2014;34(7):3663-8.

27. Kohno J, Matsui Y, Yamasaki T, Shibasaki N, Kamba T, Yoshimura K, et al. Role of mammalian target of rapamycin inhibitor in the treatment of metastatic epithelioid angiomyolipoma: a case report. Int J Urol. 2013;20(9):938-41.

28. Cibas ES, Goss GA, Kulke MH, Demetri GD, Fletcher CD. Malignant epithelioid angiomyolipoma ('sarcoma ex angiomyolipoma') of the kidney: a case report and review of the literature. Am J Surg Pathol. 2001;25(1):121-6.

29. Park HK, Zhang S, Wong MK, Kim HL. Clinical presentation of epithelioid angiomyolipoma. Int J Urol. 2007;14(1):21-5.

30. Gao J, Aksoy BA, Dogrusoz U, Dresdner G, Gross B, Sumer SO, et al. Integrative analysis of complex cancer genomics and clinical profiles using the cBioPortal. Sci Signal. 2013;6(269):pl1.

31. Forbes SA, Beare D, Boutselakis H, Bamford S, Bindal N, Tate J, et al. COSMIC: somatic cancer genetics at high-resolution. Nucleic Acids Res. 2017:45(D1):D777-D83.

32. Sharma P, Retz M, Siefker-Radtke A, Baron A, Necchi A, Bedke J, et al. Nivolumab in metastatic urothelial carcinoma after platinum therapy (CheckMate 275): a multicentre, single-arm, phase 2 trial. Lancet Oncol. 2017;18(3):312-22.

33. Borghaei H, Paz-Ares L, Horn L, Spigel DR, Steins M, Ready NE, et al. Nivolumab versus docetaxel in advanced nonsquamous non-small-cell lung Cancer. N Engl J Med. 2015:373(17):1627-39.

34. Tumeh PC, Harview CL, Yearley JH, Shintaku IP, Taylor EJ, Robert L, et al. PD-1 blockade induces responses by inhibiting adaptive immune resistance. Nature. 2014;515(7528):568-71

Ready to submit your research? Choose BMC and benefit from:

- fast, convenient online submission

- thorough peer review by experienced researchers in your field

- rapid publication on acceptance

- support for research data, including large and complex data types

- gold Open Access which fosters wider collaboration and increased citations

- maximum visibility for your research: over $100 \mathrm{M}$ website views per year

At $\mathrm{BMC}$, research is always in progress.

Learn more biomedcentral.com/submissions 